\title{
Comparaison inter-laboratoires : étalonnage des instruments volumétriques à piston
}

\author{
Boris GEYNET* \\ CT2M, Route de Lançon, Centre des Creusets, 13250 Saint-Chamas, France
}

\begin{abstract}
Résumé. En 2016, le CT2M a organisé une comparaison inter-laboratoires sur l'étalonnage des micropipettes à laquelle 32 laboratoires ont participé. Parmi eux, il y avait des laboratoires d'étalonnage (accrédités et non accrédités) mais également des laboratoires d'essais réalisant les étalonnages et/ou contrôles de leurs micropipettes en interne. Le traitement des résultats a été réalisé selon les principes statistiques de l'ISO 13528 et en respectant les exigences de l'ISO 17043. Cet article présentera l'organisation de cette comparaison inter-laboratoires ainsi que les résultats obtenus. Les performances des participants sont évaluées et une interprétation des résultats est proposée afin de mettre en évidence les paramètres d'influence prépondérants sur les résultats d'étalonnage des instruments volumétriques à piston.
\end{abstract}

\section{Introduction}

Les organismes accréditeurs demandent aux laboratoires accrédités de participer régulièrement à des comparaisons inter-laboratoires afin de prouver leur aptitude à réaliser des essais ou des étalonnages. Depuis plusieurs années, le CT2M organise des essais interlaboratoires dans des domaines variés afin de répondre à ce besoin. En 2016, une comparaison inter-laboratoires a été organisée sur l'étalonnage des pipettes à piston. 32 laboratoires ont participé à ce circuit qui s'est déroulé de Mars 2016 à Février 2017. Parmi eux, il y avait des laboratoires d'étalonnage (accrédités ou non) ainsi que des laboratoires d'essais réalisant en interne les étalonnages et/ou contrôles de leurs micropipettes.

Cet essai d'aptitude a été organisé conformément aux exigences de l'ISO 17043, le traitement des résultats et l'étude des performances des participants a été réalisé selon les principes de l'ISO 13258. Les résultats obtenus sont présentés dans cet article.

\section{Organisation du circuit}

\subsection{Entités soumises à l'essai d'aptitude}

Les instruments servant de supports de comparaison pour ce circuit étaient trois micropipettes à volume variable :

- Appareil volumétrique à piston, type A - BIOHIT 10191041 - volume variable 5-50 $\mu 1$

- Appareil volumétrique à piston, type A EPPENDORF 278993A - volume variable 20-200 $\mu$ l

- Appareil volumétrique à piston, type A - RAININ E1120249T - volume variable 100-1000 $\mu$ l.
Ces instruments ont été choisis afin de couvrir la gamme des volumes couramment utilisés dans les laboratoires. Par ailleurs, des instruments de marque et modèle différents ont volontairement été choisis de façon à être représentatif des équipements utilisés par les laboratoires.

\subsection{Protocole de réalisation des essais}

La méthode d'étalonnage préconisée était la méthode gravimétrique selon la norme ISO 8655-6, avec 10 pesées. Toutefois, toute autre méthode pouvait être utilisée par les participants ne disposant pas des moyens nécessaires pour mettre en œuvre cette méthode de référence. 31 participants ont utilisé la méthode de référence, un seul laboratoire ayant mis en œuvre une méthode colorimétrique.

Pour chacun des instruments à étalonner, les laboratoires ont dû déterminer l'erreur systématique et l'erreur aléatoire (écart-type) pour trois volumes d'essai par instrument : $5-50 \mu 1$,

$-5 \mu 1,25 \mu \mathrm{l}$ et $50 \mu \mathrm{l}$ pour la pipette à volume variable

- $20 \mu 1,100 \mu 1$ et $200 \mu 1$ pour la pipette à volume variable $20-200 \mu 1$,

- $100 \mu 1,500 \mu 1$ et $1000 \mu 1$ pour la pipette à volume variable $100-1000 \mu 1$.

Les pointes (embouts) ont été changées entre chaque volume d'essai. 6 pointes par pipette ont tout de même été mises à disposition pour chacun des participants en cas de besoin de renouveler leur étalonnage.

L'utilisation des 3 micropipettes dédiées à cette comparaison inter-laboratoires a été limitée au cours du circuit afin d'assurer leur stabilité. Une utilisation

\footnotetext{
${ }^{*}$ E-mail : bgeynet@.ct2m.fr
} 
intensive de ces instruments pouvant engendrer une instabilité et par conséquent l'impossibilité d'exploiter les résultats de manière pertinente.

\section{Méthode d'analyse des données}

\subsection{Hypothèse de normalité et tests valeurs aberrantes}

L'objectif de cet essai d'aptitude était de réaliser une évaluation des performances des laboratoires en confrontant leurs résultats. Des hypothèses ont été prises en compte pour l'analyse des données afin de rendre des conclusions cohérentes par rapport aux résultats obtenus. Conformément aux normes de référence, l'hypothèse d'une distribution normale des séries de données a été faite. Cette hypothèse a pu être vérifiée sur l'ensemble des séries exploitées. Pour cela, deux tests de normalité ont été utilisés :

- Méthode graphique (Droite de Henry)

- Méthode statistique (Shapiro-Wilk)

Le test de Grubbs a été effectué sur l'ensemble des séries de résultats traitées dans cette comparaison interlaboratoires, le mesurande étant le "volume délivré » déterminé par chacun des laboratoires participants. L'application de ce test a mis en évidence que 2 laboratoires participants ont rendu au moins un résultat aberrant sur un des 9 volumes d'essai.

\subsection{Détermination des valeurs assignées et des écart-types associés}

\subsubsection{Valeur de référence et incertitude associée}

Les valeurs de référence de l'essai d'aptitude ont été déterminées à partir des résultats d'un laboratoire de référence accrédité selon l'ISO/CEI 17025 qui a étalonné les instruments en début et en fin de circuit. La valeur de référence calculée est la moyenne des 2 résultats, son incertitude prend en compte l'incertitude du laboratoire ainsi que la stabilité de l'instrument au cours du circuit. Le tableau ci-dessous présente les valeurs de référence obtenues ainsi que leurs incertitudes associées.

Tableau 1. Valeurs de référence et incertitudes associées

\begin{tabular}{|c|c|c|c|}
\hline & & $\mathrm{x}_{\mathrm{ref}}(\mu \mathrm{l})$ & $\mathrm{U}_{\text {ref }}$ à $\mathrm{k}=2(\mu \mathrm{l})$ \\
\hline \multirow{3}{*}{ 움 } & $5 \mu \mathrm{l}$ & 4,903 & 0,053 \\
\hline & $25 \mu \mathrm{l}$ & 24,930 & 0,066 \\
\hline & $50 \mu \mathrm{l}$ & 50,04 & 0,11 \\
\hline \multirow{3}{*}{ ¿্ণ } & $20 \mu \mathrm{l}$ & 20,23 & 0,20 \\
\hline & $100 \mu \mathrm{l}$ & 100,09 & 0,25 \\
\hline & $200 \mu \mathrm{l}$ & 199,93 & 0,56 \\
\hline \multirow{3}{*}{ 음 } & $100 \mu \mathrm{l}$ & 102,1 & 1,2 \\
\hline & $500 \mu \mathrm{l}$ & 498,5 & 1,2 \\
\hline & $1000 \mu \mathrm{l}$ & 997,7 & 3,5 \\
\hline
\end{tabular}

\subsubsection{Moyenne et écart-type robustes}

La moyenne robuste $\mathrm{x}^{*}$ et l'écart-type associé $\mathrm{S}^{*}$ sont calculés selon l'algorithme A défini dans l'ISO 13528. La moyenne et l'écart-type robustes permettent notamment d'évaluer le Z-score de chacun des participants. Le tableau ci-dessous présente les résultats obtenus pour les différents volumes d'essais pour chacune des pipettes.

Table 2. Moyennes et écart-types robustes.

\begin{tabular}{|c|c|c|c|}
\hline & & $x^{*}(\mu l)$ & $\mathrm{S}^{*}(\mu \mathrm{l})$ \\
\hline \multirow{3}{*}{ 음 } & $5 \mu l$ & 4,882 & 0,086 \\
\hline & $25 \mu \mathrm{l}$ & 24,874 & 0,12 \\
\hline & $50 \mu \mathrm{l}$ & 50,019 & 0,14 \\
\hline \multirow{3}{*}{ 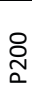 } & $20 \mu \mathrm{l}$ & 20,354 & 0,21 \\
\hline & $100 \mu \mathrm{l}$ & 100,13 & 0,50 \\
\hline & $200 \mu l$ & 200,13 & 0,65 \\
\hline \multirow{3}{*}{$\begin{array}{l}\text { 음 } \\
\text { 겸 }\end{array}$} & $100 \mu \mathrm{l}$ & 103,06 & 1,5 \\
\hline & $500 \mu l$ & 499,2 & 2,6 \\
\hline & $1000 \mu l$ & 998,9 & 3,4 \\
\hline
\end{tabular}

\section{Résultats des participants}

Les résultats des participants sont présentés pour les 3 volumes nominaux des 3 instruments $(50 \mu 1,200 \mu 1$ et $1000 \mu \mathrm{l})$ dans les tableaux et graphes suivants. Sur les graphes, le code " 0 » correspond à la valeur de référence. Les barres d'erreurs associées aux volumes délivrés correspondent aux incertitudes élargies à $\mathrm{k}=2$ (pour les participants qui les ont rendues). Les traits pleins rouges correspondent aux Erreurs Maximales Tolérées (EMT) définies dans l'ISO 8655-2.

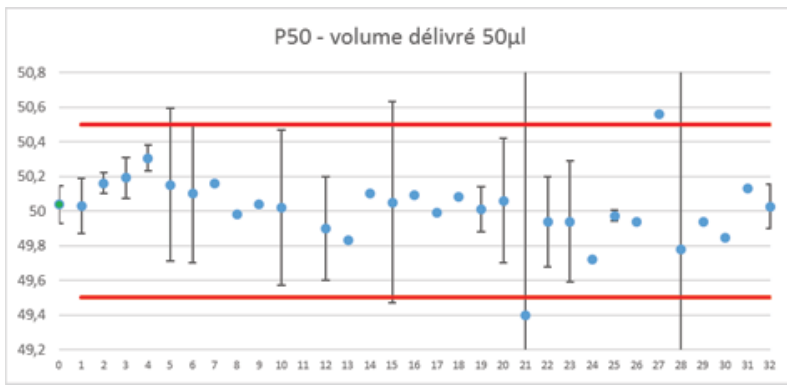

Fig. 1. Volume délivré $(50 \mu 1)$ et incertitude vs. laboratoire

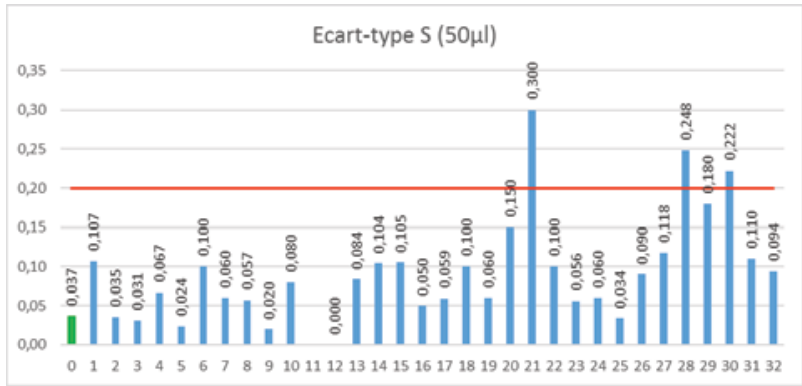

Fig. 2. Erreur aléatoire au volume $50 \mu 1$ vs. laboratoire 


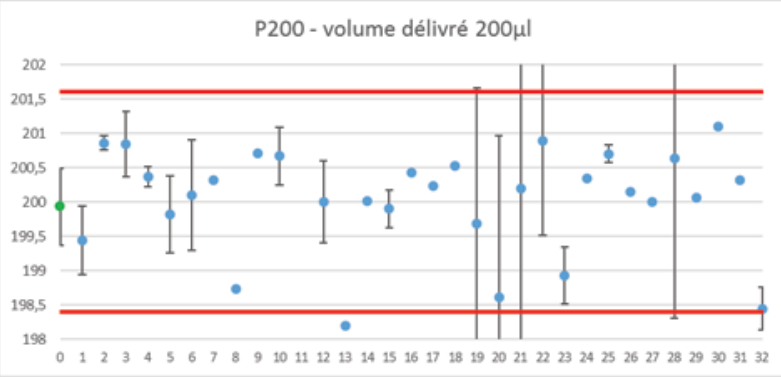

Fig. 3. Volume délivré $(200 \mu 1)$ et incertitude vs. laboratoire

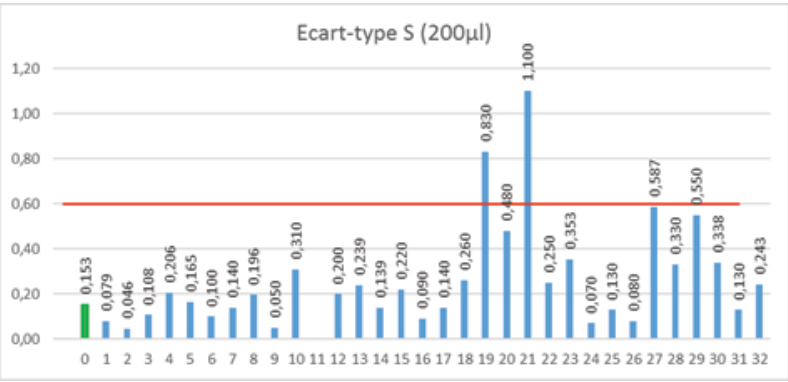

Fig. 4. Erreur aléatoire au volume $200 \mu \mathrm{l}$ vs. laboratoire

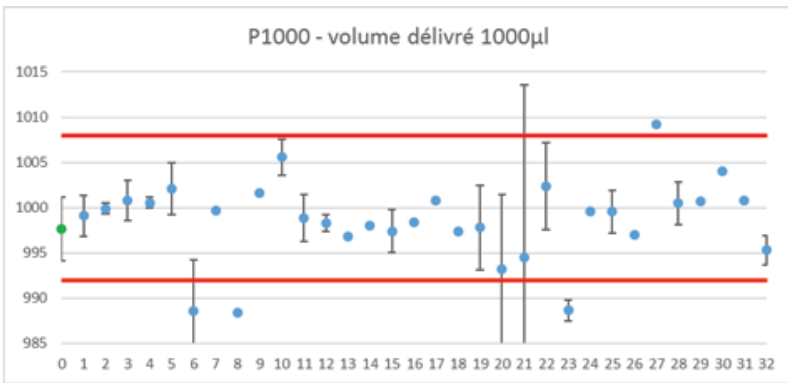

Fig. 5. Volume délivré $(1000 \mu \mathrm{l})$ et incertitude vs. laboratoire

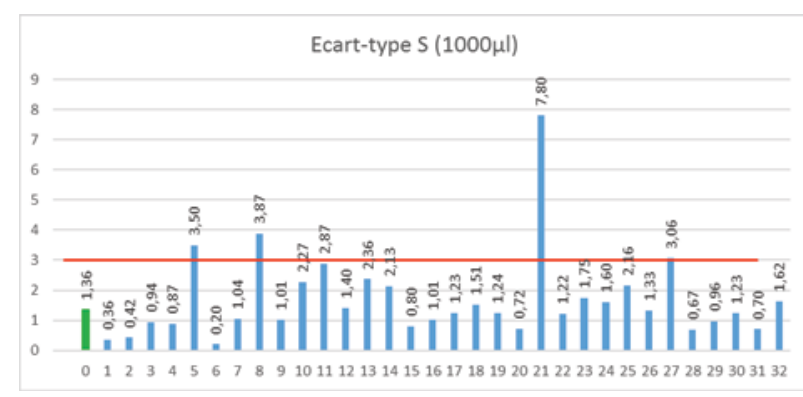

Fig. 6. Erreur aléatoire au volume $1000 \mu 1$ vs. laboratoire

\section{Performances des participants}

La performance des laboratoires a été déterminée par le Z-score qui peut être défini comme une mesure normalisée du biais. Ce score de performance est calculé selon la formule suivante :

$$
Z=\frac{\left(x_{\text {labo }}-x^{*}\right)}{S^{*}}
$$

La valeur de référence $x^{*}$ est la moyenne robuste des résultats des participants, $\mathrm{x}_{\text {labo }}$ correspond au résultat $\mathrm{du}$ laboratoire, le paramètre $S^{*}$ étant l'écart-type de l'essai d'aptitude. Les Z-scores obtenus par les participants pour les 3 volumes d'essai de la pipette $5-50 \mu 1$ sont présentés sur la figure ci-dessous.

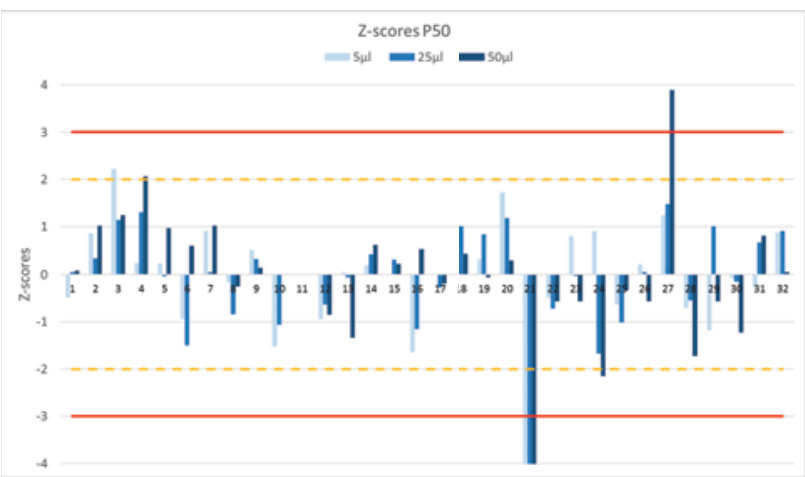

Fig. 7. Z-scores pour les 3 volumes de la pipette P50

Une inspection visuelle permet de constater que le laboratoire (21) sous-estime chacun des 3 volumes testés de la pipette P50. Par ailleurs, le laboratoire 27 surestime le volume délivré à $50 \mu 1$.

Une interprétation des résultats par rapport à la valeur de référence a également été réalisée. L'écart normalisé a été utilisé, c'est un paramètre permettant d'évaluer si la différence entre 2 valeurs est significative ou non. Pour cela, les valeurs sont comparées en prenant en compte leurs incertitudes élargies associées. Les résultats des participants sont donc comparés à la valeur de référence. L'écart normalisé est calculé selon la formule suivante :

$$
E n=\frac{\left|x_{l a b o}-x_{r e f}\right|}{\sqrt{U_{l a b c}^{2}+U_{r e f}^{2}}}
$$

Où $\mathrm{x}_{\mathrm{ref}}$ est la valeur de référence, $\mathrm{x}_{\text {labo }}$ est le résultat du laboratoire, $\mathrm{U}_{\text {ref }}$ est l'incertitude élargie $(\mathrm{k}=2)$ sur la valeur de référence, et $\mathrm{U}_{\text {labo }}$ est l'incertitude élargie $(\mathrm{k}=2)$ sur le résultat du laboratoire.

La figure ci-dessous présente les écarts normalisés pour les 3 volumes de la pipette P200, ceux-ci sont représentés uniquement pour les participants ayant estimé et rendu leurs incertitudes.

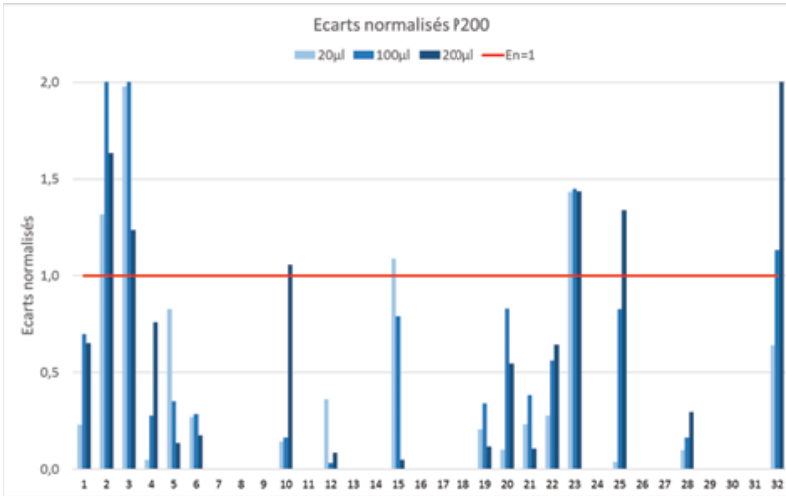

Fig. 8. Ecarts normalisés pour les 3 volumes de la P200 
L'interprétation de l'écart normalisé doit se faire avec précaution. En effet certains laboratoires ont des incertitudes importantes, ce qui entraine un écart normalisé correct (inférieur à 1) malgré un biais important par rapport à la valeur de référence (laboratoire 20 par exemple). A l'inverse, malgré un biais faible, certains laboratoires ont un écart normalisé supérieur à 1 à cause d'une incertitude sans doute sousestimée.

\section{Conclusions}

Cette comparaison inter-laboratoires réunissant un nombre de laboratoires conséquent a permis de mettre en évidence plusieurs conclusions.

Les résultats des participants ont été exploités et des critères de performances ont pu être rendus aux participants afin qu'ils puissent: soit valider leur méthode d'étalonnage comme l'exigent aujourd'hui les organismes accréditeurs, soit améliorer celle-ci en déclenchant des actions pour corriger un éventuel biais. Les résultats montrent qu'il est possible de corréler les résultats de cet essai inter-laboratoires avec la méthode d'étalonnage et les moyens mis en œuvre par chacun des laboratoires. En effet, le laboratoire ayant utilisé la méthode colorimétrique a obtenu un résultat aberrant à $50 \mu \mathrm{l}$. L'autre participant présentant au moins un résultat aberrant est le laboratoire 21 qui utilise un instrument avec une résolution relativement élevée $(0,1 \mathrm{mg})$ et une EMT de $1 \mathrm{mg}$. Par ailleurs, les participants n'ayant pas utilisé de piège à évaporation ont des résultats plus éloignés des valeurs assignées et généralement des erreurs aléatoires plus importantes.
Enfin, il est à noter que plusieurs laboratoires accrédités ont participé à cet essai inter-laboratoires et certains ont des résultats significativement différents des valeurs assignées. Une des explications est la probable sousestimation de l'incertitude et notamment de l'effet opérateur qui est une composante prépondérante sur la détermination du volume délivré par une pipette.

\section{Références}

1. NF EN ISO 8655-6 Avril 2003 : Appareils volumétriques à piston - Partie 6 : méthodes gravimétriques pour la détermination de l'erreur de mesure

2. NF EN ISO 8655-7 Février 2006: Appareils volumétriques à piston - Partie 7 : méthodes non gravimétriques pour l'estimation de la performance d'équipement

3. NF EN ISO/CEI 17043 Avril 2010 : Évaluation de la conformité - Exigences générales concernant les essais d'aptitude

4. NF ISO 13528 Octobre 2015 : Méthodes statistiques utilisées dans les essais d'aptitude par comparaisons inter-laboratoires

5. LAB GTA 90: Etalonnage des instruments volumétriques à piston (www.cofrac.fr) 